\title{
SHARP ESTIMATES OF THE POTENTIAL KERNEL FOR THE HARMONIC OSCILLATOR WITH APPLICATIONS
}

\author{
ADAM NOWAK AND KRZYSZTOF STEMPAK
}

\begin{abstract}
We prove qualitatively sharp estimates of the potential kernel for the harmonic oscillator. These bounds are then used to show that the $L^{p}-L^{q}$ estimates of the associated potential operator obtained recently by Bongioanni and Torrea are in fact sharp.
\end{abstract}

\section{$\S 1$. Introduction}

The study of the potential theory for the $d$-dimensional harmonic oscillator

$$
\mathcal{H}=-\Delta+\|x\|^{2}
$$

has recently been initiated by Bongioanni and Torrea [2]. The multidimensional Hermite functions $h_{k}$ are eigenfunctions of $\mathcal{H}$, and we have $\mathcal{H} h_{k}=$ $(2|k|+d) h_{k}$. The operator $\mathcal{H}$ has a natural self-adjoint extension, here still denoted by $\mathcal{H}$, whose spectral decomposition is given by $h_{k}$.

The integral kernel $G_{t}(x, y)$ of the Hermite semigroup $\{\exp (-t \mathcal{H}): t>0\}$ is known explicitly to be

$$
\begin{aligned}
G_{t}(x, y) & =\sum_{n=0}^{\infty} e^{-(2 n+d) t} \sum_{|k|=n} h_{k}(x) h_{k}(y) \\
& =(2 \pi \sinh (2 t))^{-d / 2} \exp \left(-\frac{1}{4}\left[\tanh (t)\|x+y\|^{2}+\operatorname{coth}(t)\|x-y\|^{2}\right]\right) .
\end{aligned}
$$

(See [6] for this symmetric variant of the formula.)

Received November 15, 2011. Accepted May 10, 2012.

First published online June 20, 2013.

2010 Mathematics Subject Classification. Primary 47G40; Secondary 31C15.

The work of both authors was partially supported by Ministry of Science and Higher Education (Poland) grant N N201 417839.

(C) 2013 by The Editorial Board of the Nagoya Mathematical Journal 
Given $\sigma>0$, consider the negative power $\mathcal{H}^{-\sigma}$, which is a contraction on $L^{2}\left(\mathbb{R}^{d}\right)$. It is easily seen that $\mathcal{H}^{-\sigma}$ coincides in $L^{2}\left(\mathbb{R}^{d}\right)$ with the potential operator

$$
\mathcal{I}^{\sigma} f(x)=\int_{\mathbb{R}^{d}} \mathcal{K}^{\sigma}(x, y) f(y) d y,
$$

where the potential kernel is given by

$$
\mathcal{K}^{\sigma}(x, y)=\frac{1}{\Gamma(\sigma)} \int_{0}^{\infty} G_{t}(x, y) t^{\sigma-1} d t .
$$

Note that all the spaces $L^{p}\left(\mathbb{R}^{d}\right), 1 \leq p \leq \infty$, are contained in the natural domain of $\mathcal{I}^{\sigma}$ consisting of those functions $f$ for which the integral in (1) converges $x$ almost everywhere (see [4, Section 2]).

The main result of this paper, Theorem 2.4, provides qualitatively sharp estimates of the potential kernel (2). As an application of this result, we prove sharpness of the $L^{p}-L^{q}$ estimates for the potential operator (1) obtained recently by Bongioanni and Torrea [2, Theorem 8] (see Theorem 3.1 below).

Recall that an operator $T$ defined on $L^{p}\left(\mathbb{R}^{d}\right)$ for some $1 \leq p \leq \infty$, with values in the space of measurable functions on $\mathbb{R}^{d}$, is said to be of weak type $(p, q), 1 \leq q<\infty$, provided that

$$
\left|\left\{x \in \mathbb{R}^{d}:|T f(x)|>\lambda\right\}\right| \leq C\left(\|f\|_{p} / \lambda\right)^{q},
$$

with $C>0$ independent of $f \in L^{p}\left(\mathbb{R}^{d}\right)$ and $\lambda>0$. The restricted weak type $(p, q)$ of $T$ means that $(3)$ holds for $f=\chi_{E}$, where $E$ is any measurable subset of $\mathbb{R}^{d}$ of finite measure. By definition, weak type $(p, \infty)$ coincides with strong type $(p, \infty)$; that is, the estimate $\|T f\|_{\infty} \leq C\|f\|_{p}, f \in L^{p}\left(\mathbb{R}^{d}\right)$. In terms of Lorentz spaces, the weak type $(p, q)$ is equivalent to the boundedness from $L^{p}\left(\mathbb{R}^{d}\right)$ to $L^{q, \infty}\left(\mathbb{R}^{d}\right)$, and the restricted weak type $(p, q)$ is characterized by the boundedness from $L^{p, 1}\left(\mathbb{R}^{d}\right)$ to $L^{q, \infty}\left(\mathbb{R}^{d}\right)$ (see $[1$, Chapter 4 , Section 4]). Strong type $(p, q)$ means, of course, the $L^{p}-L^{q}$ boundedness.

The notation $X \lesssim Y$ will be used to indicate that $X \leq C Y$ with a positive constant $C$ independent of significant quantities; we will write $X \simeq Y$ when simultaneously $X \lesssim Y$ and $Y \lesssim X$. We will also use the notation $X \simeq \simeq$ $Y \exp (-c Z)$ to indicate that there exist positive constants $C, c_{1}$ and $c_{2}$, independent of significant quantities, such that

$$
C^{-1} Y \exp \left(-c_{1} Z\right) \leq X \leq C Y \exp \left(-c_{2} Z\right)
$$

Further, in a number of places, we will use natural and self-explanatory generalizations of the " $\simeq \simeq$ " relation, for instance, in connection with certain 
integrals involving exponential factors. In such cases, the exact meaning

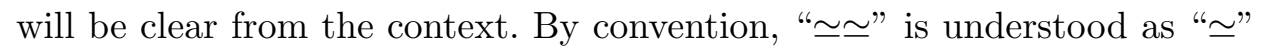
whenever no exponential factors are involved.

We write $\log ^{+}$for the positive part of the logarithm, and we write $\vee, \wedge$ for the operations of taking maximum and minimum, respectively.

\section{$\S 2$. Estimates of the potential kernel}

We begin with two technical results describing the behavior of the integrals

$$
\begin{aligned}
I_{A}(T) & =\int_{T}^{\infty} t^{A} \exp (-t) d t, \quad T>0, \\
J_{A}(T, S) & =\int_{T}^{S} t^{A} \exp (-t) d t, \quad 0<T<S<\infty .
\end{aligned}
$$

Notice that $I_{A}(T)$ dominates $J_{A}(T, S)$. The following lemma is a refinement of [4, Lemma 2.1] (see also [5, Lemma 1.1]).

Lemma 2.1. Let $A \in \mathbb{R}$ and $\gamma>0$ be fixed. Then

$$
I_{A}(\gamma T) \simeq T^{A} \exp (-\gamma T), \quad T \geq 1
$$

and for $0<T<1$,

$$
I_{A}(\gamma T) \simeq \begin{cases}T^{A+1}, & A<-1 \\ \log (2 / T), & A=-1 \\ 1, & A>-1\end{cases}
$$

Proof. We assume that $\gamma=1$. From the proof it will be clear that the estimates are true for any $\gamma>0$. The case $0<T<1$ was treated in the proof of [4, Lemma 2.1], so we consider $T \geq 1$ and focus on showing (4). The lower bound in (4) is straightforward; we have

$$
\begin{aligned}
I_{A}(T) & >\int_{T}^{2 T} t^{A} e^{-t} d t \\
& \gtrsim T^{A} \int_{T}^{2 T} e^{-t} d t=T^{A}\left(e^{-T}-e^{-2 T}\right) \gtrsim T^{A} e^{-T}, \quad T \geq 1 .
\end{aligned}
$$

It remains to prove the upper bound,

$$
\int_{T}^{\infty} t^{A} e^{-t} d t \lesssim T^{A} e^{-T}, \quad T \geq 1
$$


and here we assume that $A>0$, since for $A \leq 0$ we have $t^{A} \leq T^{A}, t>T \geq 1$, and the conclusion is trivial. Choosing $T_{A}$ such that for $T \geq T_{A}$ one has

$$
\int_{2 T}^{\infty} t^{A} e^{-t} d t \leq \frac{1}{2} \int_{T}^{\infty} t^{A} e^{-t} d t
$$

we can write

$$
\begin{aligned}
\int_{T}^{\infty} t^{A} e^{-t} d t & \leq \int_{T}^{2 T} t^{A} e^{-t} d t+\int_{2 T}^{\infty} t^{A} e^{-t} d t \\
& \leq C T^{A} e^{-T}+\frac{1}{2} \int_{T}^{\infty} t^{A} e^{-t} d t, \quad T \geq T_{A}
\end{aligned}
$$

This implies (5) for $T \geq T_{A}$ and, consequently, for all $T \geq 1$.

Lemma 2.2. Let $A \in \mathbb{R}$ and $\gamma>0$ be fixed. Then for $0<T<S \leq 2 T$ we have

$$
T^{A}(S-T) \exp (-2 \gamma T) \lesssim J_{A}(\gamma T, \gamma S) \lesssim T^{A}(S-T) \exp (-\gamma T),
$$

while for $S>2 T>0$ we have $J_{A}(\gamma T, \gamma S) \simeq I_{A}(\gamma T)$ when $S \geq 2$, and

$$
J_{A}(\gamma T, \gamma S) \simeq \begin{cases}T^{A+1}, & A<-1, \\ \log (S / T), & A=-1, \\ S^{A+1}, & A>-1,\end{cases}
$$

when $0<S<2$.

Proof. As in the proof of Lemma 2.1, it is enough to deal with the case $\gamma=1$. The bounds for $T<S \leq 2 T$ follow since then $\int_{T}^{S} t^{A} e^{-t} d t \simeq$ $T^{A} \int_{T}^{S} e^{-t} d t$ and

$$
(S-T) e^{-2 T} \leq \int_{T}^{S} e^{-t} d t \leq(S-T) e^{-T}
$$

Assume now that $S>2 T$. Clearly, $J_{A}(T, S)<I_{A}(T)$. On the other hand, if $T \geq 1$, then

$$
J_{A}(T, S)>\int_{T}^{2 T} t^{A} e^{-t} d t \gtrsim T^{A} \int_{T}^{2 T} e^{-t} d t \gtrsim T^{A} e^{-T} \gtrsim I_{A}(T),
$$

the last estimate being a consequence of (4). When $0<T<1$, we distinguish two subcases. If $S \geq 2$, then again, $J_{A}(T, S) \gtrsim \int_{T}^{2} t^{A} d t \gtrsim I_{A}(T)$. If $2 T<S<2$, then $J_{A}(T, S) \simeq \int_{T}^{S} t^{A} d t$, and evaluating the last integral, we arrive at the claimed bounds for $J_{A}(T, S)$. 
We note that (4) and (6) may be written slightly less precisely as

$$
\begin{aligned}
I_{A}(\gamma T) & \simeq \simeq \exp (-c T), \quad T \geq 1, \\
J_{A}(\gamma T, \gamma S) & \simeq T^{A}(S-T) \exp (-c T), \quad 0<T<S \leq 2 T,
\end{aligned}
$$

respectively. This fact will be used again without further mention.

We now apply Lemmas 2.1 and 2.2 to prove qualitatively sharp estimates of the integral

$$
E_{A}(T, S)=\int_{0}^{1} t^{A} \exp \left(-T t^{-1}-S t\right) d t, \quad 0<T, S<\infty .
$$

The following result provides, in particular, a refinement and generalization of [3, Lemma 2.4].

Lemma 2.3. Let $A \in \mathbb{R}$ be fixed. Then

$$
E_{A}(T, S) \simeq \simeq \exp (-c \sqrt{T(T \vee S)}) \times \begin{cases}T^{A+1}, & A<-1 \\ 1+\log ^{+} \frac{1}{T(T \vee S)}, & A=-1 \\ (S \vee 1)^{-A-1}, & A>-1\end{cases}
$$

uniformly in $T, S>0$.

Proof. We first estimate $E_{A}(T, S)$ in terms of the integrals $I_{A}$ and $J_{A}$. For $0<S \leq 2 T$, we have

$$
\begin{aligned}
E_{A}(T, S) \simeq \simeq \int_{0}^{1} t^{A} \exp \left(-c T t^{-1}\right) d t & \simeq T^{A+1} \int_{c T}^{\infty} u^{-A-2} e^{-u} d u \\
& =T^{A+1} I_{-A-2}(c T)
\end{aligned}
$$

where the second relation follows by the change of variable $t=c T / u$. When $S>2 T$, we change the variable $t=u \sqrt{T / S}$ and we get

$$
E_{A}(T, S)=\left(\frac{T}{S}\right)^{(A+1) / 2} \int_{0}^{\sqrt{S / T}} u^{A} \exp \left(-\sqrt{T S}\left(u+u^{-1}\right)\right) d u \equiv \mathcal{J}_{1}+\mathcal{J}_{2}
$$

where $\mathcal{J}_{1}$ and $\mathcal{J}_{2}$ come from splitting the integration over the intervals $(0,1)$ and $(1, \sqrt{S / T})$, respectively. Then 


$$
\begin{aligned}
\mathcal{J}_{1} \simeq\left(\frac{T}{S}\right)^{(A+1) / 2} \int_{0}^{1} u^{A} \exp \left(-c \sqrt{T S} u^{-1}\right) d u & \simeq T^{A+1} \int_{c \sqrt{T S}}^{\infty} z^{-A-2} e^{-z} d z \\
& =T^{A+1} I_{-A-2}(c \sqrt{T S})
\end{aligned}
$$

and

$$
\begin{aligned}
\mathcal{J}_{2} \simeq \simeq\left(\frac{T}{S}\right)^{(A+1) / 2} \int_{1}^{\sqrt{S / T}} u^{A} \exp (-c \sqrt{T S} u) d u & \simeq S^{-A-1} \int_{c \sqrt{T S}}^{c S} z^{A} e^{-z} d z \\
& =S^{-A-1} J_{A}(c \sqrt{T S}, c S)
\end{aligned}
$$

Summing up, we have

$$
E_{A}(T, S) \simeq \simeq T^{A+1} I_{-A-2}(c \sqrt{T(T \vee S)})+\chi_{\{S>2 T\}} S^{-A-1} J_{A}(c \sqrt{T S}, c S),
$$

uniformly in $S, T>0$. In the next step, we describe the behavior of the two terms here by means of Lemmas 2.1 and 2.2 .

From Lemma 2.1 it follows that

$$
T^{A+1} I_{-A-2}(c \sqrt{T(T \vee S)}) \simeq \simeq T^{A+1} \exp (-c \sqrt{T(T \vee S)}), \quad T(T \vee S) \geq 1
$$

(here, and also in analogous places below, $c$ on the left-hand side should be understood as a given constant) and that

$$
T^{A+1} I_{-A-2}(c \sqrt{T(T \vee S)}) \simeq \begin{cases}T^{A+1}, & A<-1, \\ \log \left(\frac{4}{T(T \vee S)}\right), & A=-1, \quad T(T \vee S) \leq 1 \\ \left(\frac{T}{T \vee S}\right)^{(A+1) / 2}, & A>-1\end{cases}
$$

The term $S^{-A-1} J_{A}(c \sqrt{T S}, c S)$ comes into play when $S>2 T$, and in this case we use Lemma 2.2 to write the bounds

$$
S^{-A-1} J_{A}(c \sqrt{T S}, c S) \simeq \chi_{\{S \geq 2\}} \Phi_{1}+\chi_{\{S<2\}} \Phi_{2},
$$

where

$$
\Phi_{1}=S^{-A-1} I_{A}(c \sqrt{T S}), \quad \Phi_{2}= \begin{cases}(T / S)^{(A+1) / 2}, & A<-1 \\ \log \left(\frac{S}{T}\right), & A=-1 \\ 1, & A>-1\end{cases}
$$


By Lemma 2.1,

$$
\begin{aligned}
& \Phi_{1} \simeq \simeq S^{-A-1} \exp (-c \sqrt{T S}), \quad T S \geq 1, \\
& \Phi_{1} \simeq \begin{cases}(T / S)^{(A+1) / 2}, & A<-1, \\
\log \left(\frac{4}{T S}\right), & A=-1, \\
S^{-A-1}, & A>-1 .\end{cases}
\end{aligned}
$$

To proceed, it is convenient to consider each of the cases $A<-1, A=-1$, and $A>-1$ separately.

If $A<-1$, then

$$
\begin{aligned}
E_{A}(T, S) \simeq \simeq & \chi_{\{2>S>2 T\}}\left(\frac{T}{S}\right)^{(A+1) / 2} \\
& + \begin{cases}T^{A+1} \exp (-c \sqrt{T(T \vee S)}), & T(T \vee S) \geq 1, \\
T^{A+1}, & T(T \vee S)<1,\end{cases} \\
& +\chi_{\{S>2 T\}} \chi_{\{S \geq 2\}} \begin{cases}T^{A+1} \exp (-c \sqrt{T S}), & T S \geq 1, \\
\left(\frac{T}{S}\right)^{(A+1) / 2}, & T S<1 .\end{cases}
\end{aligned}
$$

Here the first and third terms are insignificant compared with the second one. In case of the third summand, this is because $A<-1$ and $(T / S)^{(A+1) / 2}<$ $T^{A+1}$ for $T S<1$. A similar argument is used for the first one. The required estimates of $E_{A}(T, S)$ follow.

If $A=-1$, then

$$
\begin{aligned}
E_{-1}(T, S) \simeq \simeq & \chi_{\{2>S>2 T\}} \log \frac{S}{T}+ \begin{cases}\exp (-c \sqrt{T(T \vee S)}), & T(T \vee S) \geq 1, \\
\log \left(\frac{4}{T(T \vee S)}\right), & T(T \vee S)<1,\end{cases} \\
& +\chi_{\{S>2 T\}} \chi_{\{S \geq 2\}} \begin{cases}\exp (-c \sqrt{T S}), & T S \geq 1, \\
\log \left(\frac{4}{T S}\right), & T S<1 .\end{cases}
\end{aligned}
$$

Similar to the case of $A<-1$, here also the first and third terms are insignificant compared with the second one. This is clear for the third summand, and for the first one this is because $\log (S / T)<\log (4 /(T S))$ when $S<2$. Thus, the desired bounds of $E_{-1}(T, S)$ also follow.

Finally, we consider the case $A>-1$, which is less direct than the previous two. We have 


$$
\begin{gathered}
E_{A}(T, S) \simeq \simeq \chi_{\{2>S>2 T\}}+ \begin{cases}T^{A+1} \exp (-c \sqrt{T(T \vee S)}), & T(T \vee S) \geq 1, \\
\left(\frac{T}{T \vee S}\right)^{(A+1) / 2}, & T(T \vee S)<1,\end{cases} \\
+\chi_{\{S>2 T\}} \chi_{\{S \geq 2\}} \begin{cases}T^{A+1} \exp (-c \sqrt{T S}), & T S \geq 1, \\
S^{-A-1}, & T S<1 .\end{cases}
\end{gathered}
$$

Observe that here the relation $\simeq \simeq$ remains valid if the sum of the first and the third terms is replaced by the comparable (in the sense of $\simeq$ ) expression

$$
\chi_{\{S>2 T\}} \begin{cases}T^{A+1} \exp (-c \sqrt{T S}), & T S \geq 1 \\ (S \vee 1)^{-A-1}, & T S<1\end{cases}
$$

Taking into account that $T^{A+1} \exp (-c \sqrt{T S}) \simeq \simeq S^{-A-1} \exp (-c \sqrt{T S})$ for $T S \geq 1$, we conclude that

$$
\begin{aligned}
E_{A}(T, S) \simeq \simeq & \begin{cases}(T \vee S)^{-A-1} \exp (-c \sqrt{T(T \vee S)}), & T(T \vee S) \geq 1, \\
\left(\frac{T}{T \vee S}\right)^{(A+1) / 2}, & T(T \vee S)<1,\end{cases} \\
& +\chi_{\{S>2 T\}} \begin{cases}S^{-A-1} \exp (-c \sqrt{T S}), & T S \geq 1, \\
(S \vee 1)^{-A-1}, & T S<1 .\end{cases}
\end{aligned}
$$

Now, if $T \geq S$ and $T(T \vee S)=T^{2}<1$, then $(T /(T \vee S))^{1 / 2}=1 \simeq 1 /(S \vee$ 1), while for $T<S$ and $T(T \vee S)=T S<1$, we have $(T /(T \vee S))^{1 / 2}=$ $(T / S)^{1 / 2}<1 /(S \vee 1)$. Therefore,

$$
E_{A}(T, S) \simeq \simeq \begin{cases}(T \vee S)^{-A-1} \exp (-c \sqrt{T(T \vee S)}), & T(T \vee S) \geq 1 \\ (S \vee 1)^{-A-1}, & T(T \vee S)<1\end{cases}
$$

We claim that this implies that

$$
E_{A}(T, S) \simeq \simeq(S \vee 1)^{-A-1} \exp (-c \sqrt{T(T \vee S)}),
$$

which are precisely the required estimates.

To justify the claim, it is enough to recall that $A>-1$ and to observe that if $T \geq S$ and $T(T \vee S)=T^{2} \geq 1$, then

$$
\begin{aligned}
(T \vee S)^{-A-1} \exp (-c \sqrt{T(T \vee S)}) & =T^{-A-1} \exp (-c T) \\
& \simeq(T \vee 1)^{-A-1} \exp (-c T) \\
& \simeq \simeq(S \vee 1)^{-A-1} \exp (-c T)
\end{aligned}
$$


while if $T<S$ and $T(T \vee S)=T S \geq 1$ (this forces $S>1$ ), then

$$
\begin{aligned}
(T \vee S)^{-A-1} \exp (-c \sqrt{T(T \vee S)}) & =S^{-A-1} \exp (-c \sqrt{T S}) \\
& \simeq(S \vee 1)^{-A-1} \exp (-c \sqrt{T S})
\end{aligned}
$$

The proof is finished.

We are now in a position to prove qualitatively sharp estimates of the potential kernel.

Theorem 2.4. For $\sigma>0$, we have

$$
\begin{aligned}
\mathcal{K}^{\sigma}(x, y) \simeq \simeq \exp (-c\|x-y\|(\|x\|+\|y\|)) & \\
& \times \begin{cases}\|x-y\|^{2 \sigma-d}, & \sigma<d / 2, \\
1+\log ^{+} \frac{1}{\|x-y\|(\|x\|+\|y\|)}, & \sigma=d / 2 \\
(1+\|x+y\|)^{d-2 \sigma}, & \sigma>d / 2\end{cases}
\end{aligned}
$$

uniformly in $x, y \in \mathbb{R}^{d}$.

Proof. We decompose

$$
\begin{aligned}
\Gamma(\sigma) \mathcal{K}^{\sigma}(x, y) & =\int_{0}^{1} G_{t}(x, y) t^{\sigma-1} d t+\int_{1}^{\infty} G_{t}(x, y) t^{\sigma-1} d t \\
& \equiv \mathcal{J}_{0}^{\sigma}(x, y)+\mathcal{J}_{\infty}^{\sigma}(x, y) .
\end{aligned}
$$

For $0<t<1$, we have $\tanh t \simeq t, \operatorname{coth} t \simeq t^{-1}, \sinh 2 t \simeq t$, and therefore,

$$
\mathcal{J}_{0}^{\sigma}(x, y) \simeq \simeq E_{\sigma-d / 2-1}\left(c\|x-y\|^{2}, c\|x+y\|^{2}\right) .
$$

This combined with Lemma 2.3 shows that the estimates from the statement hold with $\mathcal{K}^{\sigma}(x, y)$ replaced by $\mathcal{J}_{0}^{\sigma}(x, y)$. Further, taking into account that $\tanh t \simeq 1 \simeq \operatorname{coth} t$ for $t>1$, we see that

$$
\mathcal{J}_{\infty}^{\sigma}(x, y) \simeq \simeq \exp \left(-c\left(\|x\|^{2}+\|y\|^{2}\right)\right) .
$$

Thus, $\mathcal{J}_{0}^{\sigma}(x, y)$ dominates $\mathcal{J}_{\infty}^{\sigma}(x, y)$ in the above decomposition, in the sense that

$$
\mathcal{J}_{\infty}^{\sigma}(x, y) \lesssim E_{\sigma-d / 2-1}\left(c\|x-y\|^{2}, c\|x+y\|^{2}\right)
$$

for a sufficiently small constant $c>0$. The conclusion follows. 


\section{$\S 3$. Sharpness of the $L^{p}-L^{q}$ boundedness of the potential operator}

Given $0<\sigma<d / 2$, define the region

$$
\begin{aligned}
R= & \left\{\left(\frac{1}{p}, \frac{1}{q}\right): 0 \leq \frac{1}{p} \leq 1 \text { and } 0 \vee\left(\frac{1}{p}-\frac{2 \sigma}{d}\right) \leq \frac{1}{q} \leq 1 \wedge\left(\frac{1}{p}+\frac{2 \sigma}{d}\right)\right\} \\
& \backslash\left(\left\{\left(\frac{1}{p}, \frac{1}{q}\right): 0 \leq \frac{1}{p} \leq 1-\frac{2 \sigma}{d} \text { and } \frac{1}{q}=\frac{1}{p}+\frac{2 \sigma}{d}\right\}\right. \\
& \left.\cup\left\{\left(\frac{2 \sigma}{d}, 0\right),\left(1,1-\frac{2 \sigma}{d}\right)\right\}\right)
\end{aligned}
$$

contained in the unit $(1 / p, 1 / q)$-square $[0,1]^{2}$ (see Figure 1 ).

The following result enhances [2, Theorem 8] (see also [4, Theorem 2.3]).

Theorem 3.1. Let $d \geq 1$, let $0<\sigma<d / 2$, and let $1 \leq p, q \leq \infty$. Then $\mathcal{I}^{\sigma}: L^{p}\left(\mathbb{R}^{d}\right) \rightarrow L^{q}\left(\mathbb{R}^{d}\right)$ boundedly if and only if $(1 / p, 1 / q)$ lies in the region $R$.

On the other hand, $\mathcal{I}^{\sigma}$ is not even of restricted weak type $(p, q)$ when $(1 / p, 1 / q)$ is not in the closure of $R$. Moreover, $\mathcal{I}^{\sigma}$ is of weak type $(p, q)$

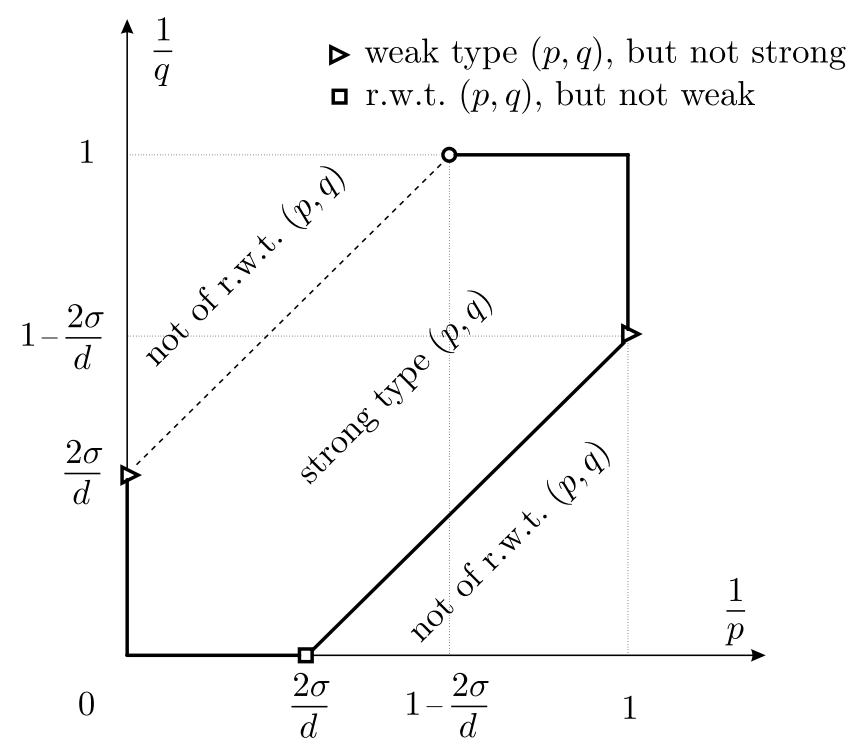

Figure 1: Mapping properties of $\mathcal{I}^{\sigma}$ for $0<\sigma<d / 2$ (r.w.t. $=$ restricted weak type). 
for $(1 / p, 1 / q)=(0,2 \sigma / d)$ and $(1 / p, 1 / q)=(1,1-2 \sigma / d)$. For $(1 / p, 1 / q)=$ $(2 \sigma / d, 0)$, the restricted weak type is true, whereas the weak type fails.

Before giving the proof, we present a short argument showing [2, (21) and (41)], the result we will apply in a moment.

Lemma 3.2. Given $\sigma>0$,

$$
\left\|\mathcal{K}^{\sigma}(x, \cdot)\right\|_{1} \simeq(1 \vee\|x\|)^{-2 \sigma}, \quad x \in \mathbb{R}^{d} .
$$

Proof. Using the identity

$$
\begin{aligned}
\exp (-t \mathcal{H}) \mathbf{1}(x) & =\int_{\mathbb{R}^{d}} G_{t}(x, y) d y \\
& =(\cosh 2 t)^{-d / 2} \exp \left(-\frac{1}{2} \tanh (2 t)\|x\|^{2}\right), \quad x \in \mathbb{R}^{d}
\end{aligned}
$$

(see [6, Proposition 3.3]), we may write

$$
\begin{aligned}
\int_{\mathbb{R}^{d}} \mathcal{K}^{\sigma}(x, y) d y & =\frac{1}{\Gamma(\sigma)} \int_{0}^{\infty} \int_{\mathbb{R}^{d}} G_{t}(x, y) d y t^{\sigma-1} d t \\
& =\frac{1}{\Gamma(\sigma)} \int_{0}^{\infty}(\cosh 2 t)^{-d / 2} \exp \left(-\frac{1}{2} \tanh (2 t)\|x\|^{2}\right) t^{\sigma-1} d t .
\end{aligned}
$$

Here we split the integration to the intervals $(0,1)$ and $(1, \infty)$ and we denote the resulting integrals by $\mathcal{J}_{0}$ and $\mathcal{J}_{\infty}$, respectively. Then, uniformly in $x \in$ $\mathbb{R}^{d}$

$$
\begin{aligned}
\mathcal{J}_{0} \simeq \simeq \int_{0}^{1} \exp \left(-c t\|x\|^{2}\right) t^{\sigma-1} d t & =\|x\|^{-2 \sigma} \int_{0}^{\|x\|^{2}} e^{-c t} s^{\sigma-1} d t \\
& \simeq\|x\|^{-2 \sigma}\left(\|x\|^{2 \sigma} \wedge 1\right)
\end{aligned}
$$

and

$$
\mathcal{J}_{\infty} \simeq \simeq \int_{1}^{\infty} e^{-t d} \exp \left(-c\|x\|^{2}\right) t^{\sigma-1} d t=C_{d, \sigma} \exp \left(-c\|x\|^{2}\right) .
$$

The conclusion follows.

Proof of Theorem 3.1. We first focus on strong-type inequalities. Then, in view of [2, Theorem 8], what remains to prove are the following two items.

(a) $\mathcal{I}^{\sigma}$ is not $L^{p}-L^{q}$ bounded for $2 \sigma / d<1 / p<1$ and $0<1 / q<1 / p-2 \sigma / d$. 
(b) $\mathcal{I}^{\sigma}$ is not $L^{p}-L^{q}$ bounded for $0<1 / p<1-2 \sigma / d$ and $1 / p+2 \sigma / d \leq$ $1 / q<1$.

To justify (a), we fix $p$ and $q$ satisfying the assumed conditions, and we define

$$
f(y)=\chi_{\{\|y\|<1\}}\|y\|^{-2 \sigma-d / q} .
$$

This function is in $L^{p}\left(\mathbb{R}^{d}\right)$ since $-(2 \sigma+d / q) p+d>0$. However, $\mathcal{I}^{\sigma} f \notin$ $L^{q}\left(\mathbb{R}^{d}\right)$. Indeed, considering $x$ such that $\|x\|<1$ and using the lower bound from Theorem 2.4, we get

$$
\begin{aligned}
\mathcal{I}^{\sigma} f(x) & \gtrsim \int_{\|y\|<\|x\| / 2}\|x-y\|^{2 \sigma-d}\|y\|^{-2 \sigma-d / q} d y \\
& \gtrsim\|x\|^{2 \sigma-d} \int_{\|y\|<\|x\| / 2}\|y\|^{-2 \sigma-d / q} d y=C\|x\|^{-d / q}
\end{aligned}
$$

and the function $x \mapsto \chi_{\{\|x\|<1\}}\|x\|^{-d / q}$ does not belong to $L^{q}\left(\mathbb{R}^{d}\right)$.

Proving (b), we may assume that $(1 / p, 1 / q)$ lies on the critical segment $1 / q=1 / p+2 \sigma / d, 0<1 / p<1-2 \sigma / d$. The case when $1 / q>1 / p+2 \sigma / d$ is contained below, in the negative result concerning the restricted weak-type estimate. Define

$$
f(y)=\chi_{\{\|y\|>e\}}\|y\|^{-d / p}(\log \|y\|)^{-1 / p-2 \sigma / d} .
$$

We have

$$
\int_{\mathbb{R}^{d}}|f(y)|^{p} d y=C_{d} \int_{e}^{\infty} r^{-1}(\log r)^{-1-2 \sigma p / d} d r<\infty,
$$

so $f \in L^{p}\left(\mathbb{R}^{d}\right)$. We claim that $\mathcal{I}^{\sigma} f \notin L^{q}\left(\mathbb{R}^{d}\right)$. Assuming that $\|x\|>2 e$ and using the lower bound from Theorem 2.4, we write

$$
\begin{aligned}
\mathcal{I}^{\sigma} f(x) \gtrsim & \int_{\|x\| / 2<\|y\|<\|x\|}\|x-y\|^{2 \sigma-d} \\
& \times \exp (-c\|x-y\|(\|x\|+\|y\|))\|y\|^{-d / p}(\log \|y\|)^{-1 / p-2 \sigma / d} d y \\
\gtrsim & \|x\|^{-d / p}(\log \|x\|)^{-1 / p-2 \sigma / d} \\
& \times \int_{\|x\| / 2<\|y\|<\|x\|}\|x-y\|^{2 \sigma-d} \exp (-2 c\|x-y\|\|x\|) d y .
\end{aligned}
$$


As we will see in a moment, the last integral is comparable with $\|x\|^{-2 \sigma}$. Thus,

$$
\begin{aligned}
\mathcal{I}^{\sigma} f(x) & \gtrsim\|x\|^{-d / p-2 \sigma}(\log \|x\|)^{-1 / p-2 \sigma / d} \\
& =\|x\|^{-d / q}(\log \|x\|)^{-1 / q}, \quad\|x\|>2 e,
\end{aligned}
$$

and the claim follows.

It remains to analyze the last integral, which we denote by $\mathcal{J}$. Changing the variable $y=x-z /\|x\|$, we get

$$
\mathcal{J}=\|x\|^{-2 \sigma} \int_{D_{x}}\|z\|^{2 \sigma-d} e^{-2 c\|z\|} d z
$$

where the set of integration is $D_{x}=\left\{z \in \mathbb{R}^{d}:\|x\|^{2} / 2<\|x\| x\|-z\|<\|x\|^{2}\right\}$. We now observe that $D_{x}$ contains the ball $B_{x}=\left\{z \in \mathbb{R}^{d}:\|x\| x\|/ 4-z\|<\right.$ $\left.\|x\|^{2} / 4\right\}$. Indeed, if $z \in B_{x}$, then

$$
\begin{aligned}
\frac{\|x\|^{2}}{2} & <\|\| \frac{x\|x\|}{4}-z\|-\| \frac{3}{4} x\|x\| \| \mid \\
& \leq\|x\| x\|-z\| \leq\left\|\frac{x\|x\|}{4}-z\right\|+\left\|\frac{3}{4} x\right\| x\|\|<\|x\|^{2} .
\end{aligned}
$$

Thus, we have

$$
\|x\|^{-2 \sigma} \int_{B_{x}}\|z\|^{2 \sigma-d} e^{-2 c\|z\|} d z \leq \mathcal{J} \leq\|x\|^{-2 \sigma} \int_{\mathbb{R}^{d}}\|z\|^{2 \sigma-d} e^{-2 c\|z\|} d z .
$$

Clearly, the integral over $\mathbb{R}^{d}$ here is finite. The integral over $B_{x}$ depends on $x$ only through $\|x\|$. Since the balls $B_{x}$ are increasing in the sense of $\subset$ when $x$ is moved away from the origin along a fixed line passing through the origin, we see that the integral over $B_{x}$ is an increasing function of $\|x\|$, which is positive and finite. We conclude that $\mathcal{J} \simeq\|x\|^{-2 \sigma},\|x\|>1$, as desired.

We pass to weak-type and restricted weak-type inequalities. Consider first the three "corners" of the boundary of $R$ from the statement of Theorem 3.1. If $(1 / p, 1 / q)=(1,1-2 \sigma / d)$, then the weak type $(1, d /(d-2 \sigma))$ holds by $[4$, Theorem 2.3]. Notice that this property can be expressed in terms of Lorentz spaces by saying that $\mathcal{I}^{\sigma}$ is bounded from $L^{1}\left(\mathbb{R}^{d}\right)$ to $L^{d /(d-2 \sigma), \infty}\left(\mathbb{R}^{d}\right)$. Then $\left(\mathcal{I}^{\sigma}\right)^{*}$ (the adjoint operator in the Banach space sense) maps boundedly 
$\left(L^{d /(d-2 \sigma), \infty}\left(\mathbb{R}^{d}\right)\right)^{*}$ into $\left(L^{1}\left(\mathbb{R}^{d}\right)\right)^{*}=L^{\infty}\left(\mathbb{R}^{d}\right)$. Further, the associate space of $L^{d /(d-2 \sigma), \infty}\left(\mathbb{R}^{d}\right)$ in the sense of $\left[1\right.$, Chapter 1 , Definition 2.3] is $L^{d /(2 \sigma), 1}\left(\mathbb{R}^{d}\right)$ (see [1, Chapter 4, Theorem 4.7]), and by [1, Chapter 1, Theorem 2.9] it can be regarded as a subspace of the dual of $L^{d /(d-2 \sigma), \infty}\left(\mathbb{R}^{d}\right)$. Since $\left(\mathcal{I}^{\sigma}\right)^{*}=\mathcal{I}^{\sigma}$ by symmetry of the kernel, we infer that $\mathcal{I}^{\sigma}$ is of restricted weak type $d /(2 \sigma), \infty$. On the other hand, the weak type $d /(2 \sigma), \infty$ coincides, by definition, with the strong type, so $\mathcal{I}^{\sigma}$ is not of weak type $d /(2 \sigma), \infty$ in view of the strong-type results we already know. This clarifies the situations related to the "corners" $(1,1-(2 \sigma / d))$ and $2 \sigma / d, 0$.

Taking into account $(1 / p, 1 / q)=(0,2 \sigma / d)$, we will show that $\mathcal{I}^{\sigma}$ is of weak type $(\infty, d /(2 \sigma))$. To do that, it is enough to verify the estimate

$$
\left|\left\{x \in \mathbb{R}^{d}:\left|\mathcal{I}^{\sigma} f(x)\right|>\lambda\right\}\right| \lesssim\left(\frac{\|f\|_{\infty}}{\lambda}\right)^{d /(2 \sigma)}, \quad \lambda>0, f \in L^{\infty}\left(\mathbb{R}^{d}\right) .
$$

But this is immediate in view of the bound (see Lemma 3.2)

$$
\left\|\mathcal{K}^{\sigma}(x, \cdot)\right\|_{1} \leq C\|x\|^{-2 \sigma}, \quad x \in \mathbb{R}^{d}
$$

since then it follows that $\left|\mathcal{I}^{\sigma} f(x)\right| \leq C\|x\|^{-2 \sigma}\|f\|_{\infty}$ and, consequently,

$$
\left\{x \in \mathbb{R}^{d}:\left|\mathcal{I}^{\sigma} f(x)\right|>\lambda\right\} \subset\left\{x \in \mathbb{R}^{d}:\|x\|<\left(C \frac{\|f\|_{\infty}}{\lambda}\right)^{1 /(2 \sigma)}\right\} .
$$

This inclusion leads directly to (7).

Finally, we disprove the restricted weak type in the two triangles (see Figure 1). In the lower triangle we use an au contraire argument involving an extension of the Marcinkiewicz interpolation theorem for Lorentz spaces due to Stein and Weiss (see [1, Chapter 4, Theorem 5.5]). Indeed, if $\mathcal{I}^{\sigma}$ were of restricted weak type $(p, q)$ for some $p$ and $q$ such that $1 / q<1 / p-$ $2 \sigma / d$, then by interpolation with a strong-type pair satisfying $1 / q=1 / p-$ $2 \sigma / d, p>1, q<\infty, \mathcal{I}^{\sigma}$ would be of strong type $(\widetilde{p}, \widetilde{q})$ for some $\widetilde{p}$ and $\widetilde{q}$ corresponding to a point in the lower triangle, a contradiction with $(a)$ above.

To treat the upper triangle, we will give an explicit counterexample. Let for large $r$

$$
f_{r}(y)=\chi_{\{\|y\|<r\}} .
$$


Clearly, we have $\left\|f_{r}\right\|_{p} \simeq r^{d / p}$. Estimating as in the proof of (b) above, we get

$$
\begin{aligned}
\mathcal{I}^{\sigma} f_{r}(x) \gtrsim & \int_{\|x\| / 2<\|y\|<\|x\|}\|x-y\|^{2 \sigma-d} \\
& \times \exp (-c\|x-y\|(\|x\|+\|y\|)) \chi_{\{\|y\|<r\}} d y \\
\geq & \chi_{\{\|x\|<r\}} \int_{\|x\| / 2<\|y\|<\|x\|}\|x-y\|^{2 \sigma-d} \exp (-2 c\|x-y\|\|x\|) d y \\
\gtrsim & \chi_{\{1<\|x\|<r\}}\|x\|^{-2 \sigma},
\end{aligned}
$$

uniformly in large $r$ and $x \in \mathbb{R}^{d}$. Consequently,

$$
\left|\left\{x \in \mathbb{R}^{d}: \mathcal{I}^{\sigma} f_{r}(x)>\lambda\right\}\right| \geq\left|\left\{1<\|x\|<r:\|x\|<(C \lambda)^{-1 /(2 \sigma)}\right\}\right|
$$

for some $C>0$ independent of $r$ and $\lambda>0$. Taking $\lambda=r^{-2 \sigma}$, we conclude that the weak-type $(p, q)$ estimate for $\mathcal{I}^{\sigma}$ implies that $r^{d} \lesssim r^{d q / p+2 \sigma q}$. This bound, however, fails when $1 / q>1 / p+2 \sigma / d$ and $r \rightarrow \infty$.

The proof is finished.

For completeness, we remark that in the context of Theorem 3.1, the question of weak/restricted weak type $(p, q)$ inequalities related to the segment $1 / q=1 / p+2 \sigma / d, 1 \leq q<2 \sigma / d$, is more subtle and remains open. Considering the case $\sigma>d / 2$, the operator $\mathcal{I}^{\sigma}$ is bounded from $L^{p}\left(\mathbb{R}^{d}\right)$ to $L^{q}\left(\mathbb{R}^{d}\right)$ for every $1 \leq p, q \leq \infty$ (see [4, Theorem 2.3]). The behavior of $\mathcal{I}^{\sigma}$ in the limiting case $\sigma=d / 2$ is described by the theorem below. This result enhances [4, Theorem 2.3] when $\sigma=d / 2$.

TheOREM 3.3. Let $d \geq 1$, and let $1 \leq p, q \leq \infty$. Then $\mathcal{I}^{d / 2}$ is bounded from $L^{p}\left(\mathbb{R}^{d}\right)$ to $L^{q}\left(\mathbb{R}^{d}\right)$ except for $(p, q)=(\infty, 1)$ and $(p, q)=(1, \infty)$. Considering the two singular cases, we have the following.

(i) $\mathcal{I}^{d / 2}$ is of weak type $(\infty, 1)$ but not of strong type $(\infty, 1)$.

(ii) $\mathcal{I}^{d / 2}$ is not of restricted weak type $(1, \infty)$.

Proof. The $L^{p}-L^{q}$ boundedness is contained in [4, Theorem 2.3]. To show (i), we observe that the weak type $(\infty, 1)$ holds true since the proof of $(7)$ covers also the case $\sigma=d / 2$. The strong type $(\infty, 1)$ fails because $\mathcal{I}^{d / 2} \mathbf{1} \notin$ $L^{1}\left(\mathbb{R}^{d}\right)$, as easily seen by means of Lemma 3.2 . 
It remains to verify (ii). For $0<\varepsilon<1 / e$, let $f_{\varepsilon}(x)=\chi_{\{\|x\|<\varepsilon\}}$. By the lower bound of Theorem 2.4, it follows that

$$
\mathcal{I}^{d / 2} f_{\varepsilon}(x) \gtrsim \int_{\|y\|<\varepsilon} \log \frac{1}{\|x-y\|(\|x\|+\|y\|)} d y, \quad\|x\|<1 / e,
$$

uniformly in $\varepsilon<1 / e$. Therefore,

$$
\begin{aligned}
\left\|\mathcal{I}^{d / 2} f_{\varepsilon}\right\|_{\infty} & \gtrsim \int_{\|y\|<\varepsilon}-\log \|y\| d y \\
& =C_{d} \int_{0}^{\varepsilon}-r^{d-1} \log r d r \gtrsim \varepsilon^{d} \log \frac{1}{\varepsilon}, \quad 0<\varepsilon<1 / e,
\end{aligned}
$$

and we conclude that

$$
\frac{\left\|\mathcal{I}^{d / 2} f_{\varepsilon}\right\|_{\infty}}{\left\|f_{\varepsilon}\right\|_{1}} \gtrsim \log \frac{1}{\varepsilon}, \quad 0<\varepsilon<1 / e .
$$

Letting $\varepsilon \rightarrow 0^{+}$, we see that $\mathcal{I}^{d / 2}$ is not of restricted weak type $(1, \infty)$.

\section{REFERENCES}

[1] C. Bennett and R. Sharpley, Interpolation of Operators, Pure Appl. Math. 129, Academic Press, Boston, 1988. MR 0928802.

[2] B. Bongioanni and J. L. Torrea, Sobolev spaces associated to the harmonic oscillator, Proc. Indian Acad. Sci. Math. Sci. 116 (2006), 337-360. MR 2256010. DOI 10.1007/ BF02829750.

[3] A. Nowak and K. Stempak, Riesz transforms and conjugacy for Laguerre function expansions of Hermite type, J. Funct. Anal. 244 (2007), 399-443. MR 2297030. DOI 10.1016/j.jfa.2006.12.010.

[4] - Negative powers of Laguerre operators, Canad. J. Math. 64 (2012), 183-216. MR 2932174. DOI 10.4153/CJM-2011-040-7.

[5] K. Stempak and J. L. Torrea, Poisson integrals and Riesz transforms for Hermite function expansions with weights, J. Funct. Anal. 202 (2003), 443-472. MR 1990533. DOI 10.1016/S0022-1236(03)00083-1.

[6] - BMO results for operators associated to Hermite expansions, Illinois J. Math. 49 (2005), 1111-1131. MR 2210354.

Adam Nowak

Instytut Matematyczny

Polska Akademia Nauk

Śniadeckich 8

00-956 Warszawa, Poland

Adam. Nowak@impan.pl 
Krzysztof Stempak

Instytut Matematyki i Informatyki

Politechnika Wroctawska

Wyb. Wyspiańskiego 27

50-370 Wroctaw, Poland

and

Katedra Matematyki i Zastosowań Informatyki

Politechnika Opolska

Mikolajczyka 5

45-271 Opole

Poland

Krzysztof.Stempak@pwr.wroc.pl 